\title{
Clarification of the Hall effect as an energy transfer mechanism in a theory of the Earth's magnetic field and sunspots
}

\begin{abstract}
A. de Paor
Abstract. Clarification is offered of the energy transfer role played by the Hall effect in a recent paper: de Paor, A., A theory of the Earth's magnetic field and of sunspots, based on a self-excited dynamo incorporating the Hall effect, Nonlinear Processes in Geophysics, 8, 265-279, 2001.
\end{abstract}

Department of Electronic and Electrical Engineering, National University of Ireland, Dublin, Belfield, Dublin 4, Ireland

Received: 26 June 2002 - Revised: 10 February 2003 - Accepted: 11 February 2003

\section{Introduction}

In a recent paper on the Earth's magnetic field and sunspots (de Paor, 2001) a key role in deriving equations governing the proposed underlying mechanism was played by the claim that the Hall effect provides "a perfect orthogonal axis energy transfer mechanism." The author has now realised that in his presentation (in Sect. 2) the meaning of this phrase is very obscure and likely to lead to misconceptions. In Eqs. (1) and (3) of de Paor (2001), two voltages $e_{z}$ and $e_{x}$ were defined. It was not made clear that these are open-circuit Hall voltages: $e_{z}$ would be measured if $i_{z}$ were zero and $e_{x}$ if $i_{x}$ were zero. When both currents are flowing, the voltages measured across the faces of the Hall Sample are modified to

$u_{x}=e_{x}+r_{x} \cdot i_{x}$

$u_{z}=e_{z}-r_{z} \cdot i_{z}$,

as will be proved below.

The voltages defined by Eq. (1) are indicated on the twoport network representation in Fig. 1 in this note. This shows that power

$p_{e}=e_{x} \cdot i_{x}$

is being extracted from the $x$-loop through the controlled source $e_{x}$ and power

$p_{i}=e_{z} \cdot i_{z}$

is being injected into the $z$-loop through the controlled source $e_{z}$.

Correspondence to: A. de Paor (annraoi.depaor@ucd.ie)

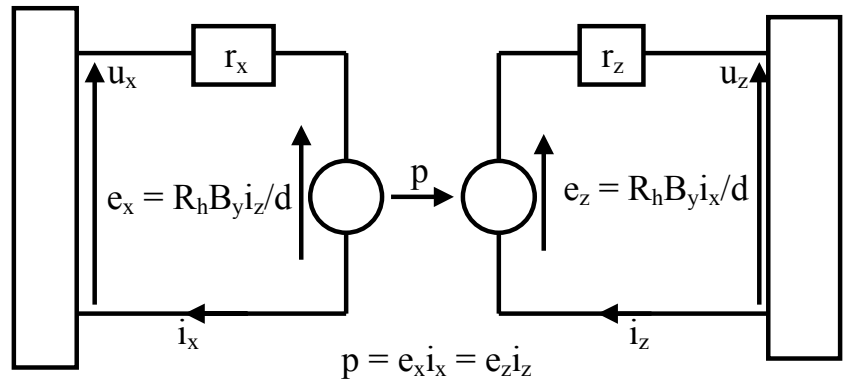

Fig. 1. Hall Sample for derivation of twoport.

Using the expressions given by de Paor (2001), we have

$p_{e}=\left(R_{h} B_{y} i_{z} / d\right) \cdot i_{x}$

and

$p_{i}=\left(R_{h} B_{y} i_{x} / d\right) \cdot i_{z}$.

It is clear that

$p_{e}=p_{i}=p$,

as indicated on Fig. 1 of this note. The power being extracted non-resistively from the $x$-loop is being injected into the $z$ loop. This, and this only, is the sense in which the Hall effect is claimed to be "a perfect orthogonal axis energy transfer mechanism".

\section{Derivation of the twoport representation in Eq. (1)}

Equation (1) is actually a macroscopic reflection of the Lorentz expression for the force on a charge carrier moving under orthogonal electric and magnetic fields in a material medium. Just for mathematical simplicity, the derivation is given here for positive charge carriers, but the author will be very happy to supply the derivation for a two-carrier conduction process, positive and negative, to anyone who may be interested. 


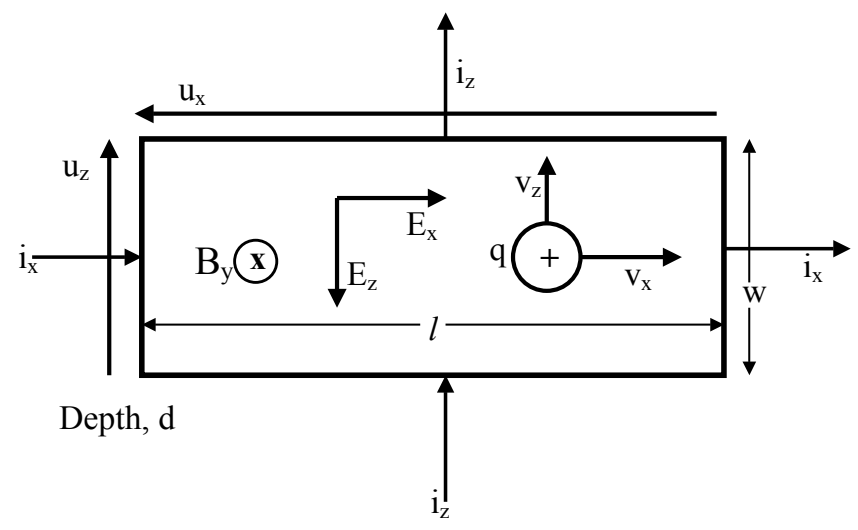

Fig. 2. Hall Sample showing cross-axis power transfer.

Figure 2 shows a Hall sample, with indicated dimensions, carrying currents $i_{x}$ and $i_{z}$ along two orthogonal axes, with a magnetic field of flux density $B_{y}$ along an axis orthogonal to them. It is assumed that the associated current densities are uniform:

$j_{x}=i_{x} /(w d)$

$j_{z}=i_{z} /(l d)$.

If the carrier concentration is $p$, if each has charge $q(\mathrm{q}>0)$ and is drifting along the $x$-axis with speed $v_{x}$ and along the $z$-axis with speed $v_{z}$, the current densities in Eq. (7) are given by

$j_{x}=q p v_{x}$

$j_{z}=q p v_{z}$.

If the mobility of a charge carrier is $\mu$ and if it experiences a force vector $\boldsymbol{F}$, then its vector drift velocity is

$\boldsymbol{v}=(\mu / q) \boldsymbol{F}$.

The force on a charge carrier along the $x$-axis is $q E_{x}$ due to the $x$-axis electric field and $-q v_{z} B_{y}$ due to the $z$-axis magnetic field. Thus, applied along the $x$-axis, Eq. (9) gives

$v_{x}=(\mu / q)\left\{q E_{x}-q v_{z} B_{y}\right\}$.

Due to the vector relationship between $v_{z}$ and $B_{y}$, the magnetic force is in the negative $x$-direction.

Multiplying Eq. (10) through by the sample length $l$, rearranging and noting that the voltage $u_{x}$ is given by $u_{x}=l E_{x}$, gives the expression

$u_{x}=r_{x} i_{x}+\left(B_{y} i_{z}\right) /(q p d)$,

where

$r_{x}=l /(\mu q p d w)$,

is the resistance of the sample in the $\mathrm{x}$-direction.

Noting that for a single species of carrier, the Hall coefficient is given by

$R_{h}=1 /(q p)$. and that

$e_{x}=R_{h} B_{y} i_{z} / d$

Equation (11) immediately becomes identical with the first member of Eq. (1). This establishes the validity of the input loop of the twoport.

If the same type of analysis is applied along the $z$-axis, the only essential difference, due to the vector relationship between $i_{x}$ and $B_{y}$, is that the magnetic force is in the positive $y$-direction. This is reflected in the equation

$u_{z}=-r_{z} i_{z}+\left(B_{y} i_{x}\right) /(q p d)$

where

$r_{z}=w /(\mu q p d l)$

is the resistance in the $z$-direction. Equation (14) immediately gives the second member of Eq. (1), which describes the output loop of the twoport.

\section{Application to geomagnetic and sunspot theory}

The twoport network in Fig. 1 here is implicit in Eqs. (11) and (12) of de Paor (2001).

In Eq. (11) are made the substitutions $i_{z} \rightarrow i_{a}, r_{z} \rightarrow$ $R_{a}, e_{z} \rightarrow e_{h a}=k_{h} i_{a} i_{d}$. The right hand box in Fig. 1 now contains the inductor $L_{a}$ in series with the Johnson-Nyquist noise voltage source of emf $n_{a}(t)$.

In Eq. (12) are made the substitutions $i_{x} \rightarrow i_{d}$ (the dynamo current), $r_{x} \rightarrow R_{d a}$ (as defined by Eq. (48) of de Paor (2001)), $e_{x}=e_{h d}=k_{h} i_{a}^{2}$. The left hand box in Fig. 1 now contains the series combination of the dynamo emf, $k_{d} i_{a}^{2}$, the resistances $R_{d r}$ and $R_{d m}$ defined by Eqs. (49) and (50) of de Paor (2001), the inductor $L_{d}$ and the Johnson-Nyquist source of emf $n_{d}(t)$.

Equations (11) and (12) of de Paor (2001) are the only places in the theory in which Eq. (6) of this note is invoked.

The author wishes to apologise to anybody who was confused by the obscurity of his original exposition. He wishes to thank Professor J.E. Allen of the University of Oxford for pointing out the obscurity.

In conclusion, a few typographical errors in de Paor (2001) are pointed out. In the second last line of page 269, the conductivity estimate should read $\sigma \approx 10^{7} \mathrm{~S} \mathrm{~m}^{-1}$. In Eq. (50), the argument of the arcsin function - which must, of course, have magnitude $\leq 1-$ should read $r_{2} / r_{3}$. In Eq. (56), in the second term within the parentheses, the square root sign should not extend over the quantity $-l / 2$. There is a multiplicative factor of $\pi$ omitted from the expression for $L_{a}$ in Eq. (57).

\section{References}

de Paor, A.: A theory of the Earth's magnetic field and of sunspots, based on a self-excited dynamo incorporating the Hall effect, Nonlinear Processes in Geophysics, 8, 265-279, 2001. 